\title{
UIPLANTS: A Software Program for the Landscape Industry and Horticulture Education
}

Gary J. Kling ${ }^{1}$, Christopher P. Lindsey², and M ark E. Zampardo ${ }^{1}$

\begin{abstract}
Summary. U IPLANTS is a computer-based reference to help identify, culture, and use woody landscape plants for the northern and central U nited States. The program provides a comprehensive reference to serve the educational and professional communities with more than $\mathbf{8 0 0 0}$ high-quality color images and textual descriptions of more than $\mathbf{9 0 0}$ species and cultivars. Special features include a highly flexible slide show and the ability to compare any two images side by side. Student activity can be tracked, creating detailed logs of student use patterns and times.
\end{abstract}

Additional IndeX words. computer, computer-aided instruction, MS Windows, trees, shrubs, vines, plants

tudents, educators and landscape professionals require visual materials to help learn, teach, and sell plant materials. In an ideal situation, live plant specimens would al ways be available, but there are many situations when this is not feasible. For example, it would not be convenient to bring live plant material to a potential customer's home. Even if live materials were available, the many seasonal features of the plant could not be seen at one time. H orticulture students often find the need to study plant materials at every stage of development at any time of the year and at all times of the day or night.

Printed landscape plant references offer only a limited number of line drawings (D irr, 1990), black and white photos (Flint, 1983; Wyman, 1990) or color images for a portion of the plants they cover due to the high costs of printing. Publications that do feature color images often are limited to presenting only one or two plant features (Phillips, 1978; Phillips and Rix, 1989) or may have only limited text to accompany the images. Even with an extensive set of reference books, students and professionals find that much of the visual plant information they need is unavailable.

Computer-based systems offer the ability to store large quantities of visual images in a compact and affordable format. This is especially true with compact disc (CD-ROM) technology, which can store up to 700 megabytes of data per disc.

\footnotetext{
${ }^{1} \mathrm{D}$ epartment of $\mathrm{N}$ atural R esourcesand Environmental Sciences, U niversity of I llinois, U rbana, I L 61801. Towhom reprint requestsshould be addressed.

${ }^{2}$ Graduateassistant, D epartment of N atural R esourcesand Environmental Sciences, U niversity of IIlinois, U rbana, IL 61801.

${ }^{3} \mathrm{C}$ oordinator of $\mathrm{H}$ orticulture, College of Lake County, Grayssiake, IL 61030.
} 
These discs can be produced at costs ranging from about $\$ 75$ each to less than $\$ 1$, depending on the quantity produced.

There are several other multimedia plant material products on the market. Southern Trees, an expert system for selecting trees (second edition), and Florida Plant Selector, from the U niv. of Florida, serve as a comprehensive guide to the ornamental features and selection of trees and shrubs from the southern U nited States. Plantit CD !, al so from the U niv. of Florida, features 1000 photographs of U.S. plants, with one picture per plant. $H$ orticopia, D esops LTD, Purceville Va., is a two-CD set, with one CD featuring perennials and annuals and another covering trees, shrubs and groundcovers. I n general, each of the $\mathrm{H}$ ortico pia disks has two or three images per plant. The Pro Series Plant D atabase for CD RO M , Green Thumb Software, Boulder Colo., has 2300 U .S. plants, with color photos and the ability to interact with landscape design software or print color photos. It has audio scientific name pronunciation.

Although the above products present images of major ornamental features nicely, and in some cases offer plant selection utilities, thecurrent productson the market do not have an extensive photographic library for any single species and do not allow side-by-side comparisons of their images. These productsusually do not show leaf, bud, stem, and bark features or other plant features such as flower, fruit, or fall color unless they are particularly showy. UIPLANTS displays text and images simultaneously to provide a greater understanding of plant features. O ther CD-ROM-based products that we have seen present either images or text on the screen, but not both, for most portions of their program.

The Dept. of $\mathrm{H}$ orticulture at the $U$ niv. of Illinoishasproduced a software program called UIPLANTS to provide descriptive information and images for woody landscape plant identification, ornamental features, and culturefor thenorthern and central $U$ nited States. D esigned to serve college students and industry professionals, U I PLAN TS takes advantage of computer and CD-ROM technology to storeand display color images in a coordinated format with text by using the interactivity that this equipment makes possible. include

Educational objectives for this program

- $\quad$ serving as a comprehensive reference to help identifiy, culture, and use woody landscape plants,

- enhancing retention of course material through increased use of visual study techniques,

- facilitating individualized instruction, and

- increasing student access to information that is not available in the classroom or not readily available from standard references. $O$ bjectives for professionals include

- serving as an informational display tool for clientele of botanic gardens, arbo reta, and retail sales establishments,

- serving as a sales tool by facilitating customized sales presentations,

- creating a program that is capable of updating by the user, and

- providing a framework program that can be used for other commodity areas.

As teachers of plant materials courses, we found that our campus grounds lacked a sufficient diversity of plant material specimens and students lacked quality visual references when studying for examinations. UIPLANTS is designed to supplement other methods of information delivery for plant materialsstudentsbut not replace laboratories where students still need to see, touch, and smell plants. Although this program could replace traditional slidelecture information delivery, it is intended to be a study tool for students preparing for laboratory or lecture examinations. The program is particularly useful when plants are not easily available or when the weather or light conditions do not permit outdoor viewing. UIPLANTS also provides individualized instruction at a pace appropriate to each student or client, allowing the user to choose the path and order of the subject matter. O ptimally, the program should be available to students at any time of the day or night and in a location convenient for their study.

Garden centers, retail nurseries, landscape designers, botanic gardens, and arboreta all have clientele who want plant material information. This program can serve as a reference in support of salespeople and also be used by knowledgeable consumers. N ew employees working sales lots often do not have thenecessary knowledgeto answer many of the questions posed by potential customers, such as "H ow big will it get? What do its flowers and fruit look like? $\mathrm{H}$ ow is it different from other species? What major problemscan I expect?" M any years of experience usually are required to develop the competency to answer some of thesequestions. Theencyclopedic portion of UIPLANTS allows for easy accessto answers for many of these questions.

Sales of landscape designs hinge on the ability of the designer to impress the customer with the beauty and functionality of the plants that have been selected for them. Because consumers usually want to see what they are getting before they agree to spend their money, designers often find themselves having to work with a number of pictorial references to illustrate plant features. I n many cases, the imagesarenot available in reference books. UIPLAN TS provides many images in one easily accessible so urce. A customized list 
feature allows users to put together a completely customized presentation. The program shows images of plants in the landscape and has text concerning landscape use, but it does not offer an extensive series of images showing functionality, massing, or other design characteristics.

Because it is easy to upgrade, owners of this software also can add their own text and images to the program to keep up to datewith new cultivars or inventory.

\section{System requirements}

UIPLAN TS can be run on nearly every new computer with either a D O S-Windows or OS/ 2 operating system.

$M$ inimum requirements are (with preferred configurations in parentheses)

- A $25 \mathrm{M} \mathrm{Hz} 486$ or $33 \mathrm{M} \mathrm{Hz} 3865 X$ (33 $\mathrm{M} \mathrm{H}$ z 486D X or better)

- 4 megabytes RAM (8 megabytes RAM)

- $\quad$ SVGA video card or equivalent with 1 megabyte VRAM (must support $1024 \times 768$ with 256 colors)

- M ouse or other pointing device

- About 5 megabytes of hard drive space for the program

- a CD-ROM drive or about 700 megabytes of disk space for the images

- Microsoft Windows 3.1 with the Arial font installed.

The program can run under several different hardware configurations. O n our development machine we store the program, images, and text on a 1-gigabyte hard drive. Most individual users with less hard drive space will operate from a CD-ROM reader and 5 megabytes of hard drive space. In this configuration, theimageswould bestored on the $C D$, and the program, including text, would be on the hard drive. Because the software is set up to look for images on multiple drives, user-supplied images on a hard drive can be accessed easily by the program.

The program also can be run from a central server over a local area network. O ur program currently runs on an $\mathrm{OS} / 2$ server at the Univ. of Illinois in the College of Agriculture's microcomputer $L$ aboratory. In this configuration, one copy of the program ran from a server that could be accessed from any of 50 individual machines. The speed of the server and the network-linked machines was sufficient to produce no noticeable delay with 10 to 15 students working on the program simultaneously.

\section{Images and text}

UIPLANTS is written in a completely modular format and contains detailed images and text for more than 900 species and cultivars of woody landscape plantsspanning more than 150 genera. All data from the program are kept in plain text files, making plant additions and deletions as simple as editing one of these text files or copying a new image to the hard drive.

I mages come from the slide collections of the authors, with additional images from Floyd Giles and $D$ ave Williams at the U niv. of Illinois and Paul Cappiello at the U niv. of $M$ aine. Slide images were scanned with a Kodak RFS 2035 film scanner, which converts the images into $480 \times 320$ Windows device-independent bitmap files (.D IB/ .BM P) with a unique palette of 256 colors. I mages are assigned DOS file names using the first three letters of the genus, the first two of the specific epithet and one letter for the cultivar, if applicable, along with a two-digit number designating the plant featureand imagenumber. With this file-naming scheme, up to 10 characteristicscan be displayed per plant with up to 10 images within each of those characteristics. This makes it possible for a plant to have 100 images associated with it, and another 100 for each of its cultivars.

Textual information and imagesareavailable on plant habit, leaves, buds, stems, flowers, fruit, fall color, cultural requirements, diseases and insects, and landscape use. In addition, information isalso available on plant size, color, texture, winter hardiness, and native range. The text was composed initially by the individual authors and then reviewed by the other authors. Additional reviewers and text contributors include D.J. Williams and F.A. Giles. Univ. of Illinois. and P. Cappiello, $U$ niv. of $M$ aine. The authors also anticipate an external review of the program and text before release.

An important feature of UIPLANTS is its ease of upgrading. The program allows for the addition of images and text for a genus, species, or cultivar by copying a properly named file to the appropriate subdirectory or by editing existing files. As a standardizing mechanism, asimpletext-formatting language similar to the $\mathrm{H}$ yperT ext $\mathrm{M}$ arkup $\mathrm{L}$ anguage (HTML) and Rich Text Format (RTF) was developed. $\mathrm{N}$ ew images can be added to the program by users, if they have access to a film scanner, by applying the naming convention described above and placing the file in the appropriate sub-directory, making them immediately available to the program without any further work. The program also has a simple utility to add text and images for new species or cultivars.

\section{Program features}

Opening screen. The opening screen of UIPLANTS allows the user to obtain information about the program, authors, and version update notes or to enter the main body of the program. O ptionally, it can serve as a place for 


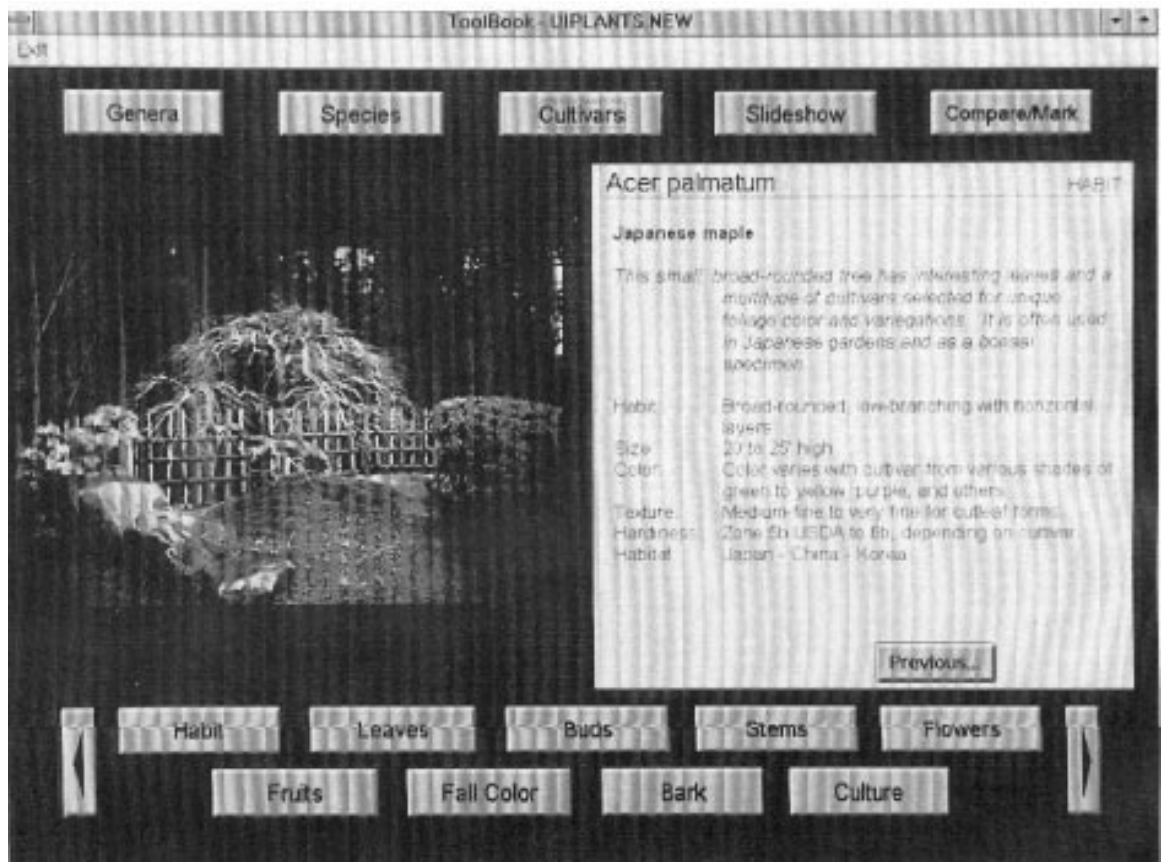

Fig. 1. Sample screen displaying information provided when a species is selected.

students to log into the program so that their efforts can be monitored. The login system prompts users for an identification code that is used by the computer to log the user's activities. When a proper code is entered, the program can be accessed. We currently are using the login system to evaluate the educational benefits of the program and the stu- dent use patterns of variousprogram features. UIPLANTS has extensive logging capabilities that list every activity performed by each user, the amount of time spent to do the activity, and the order in which activities were performed. Because these files are long and difficult to read, several programs have been developed in Perl, a language suited for parsing text files, to summarize user activitiesinto a more readable form. L ogin security can be toggled either on or off, with different degrees of activity-logging.

Main PROGRam BOdY.

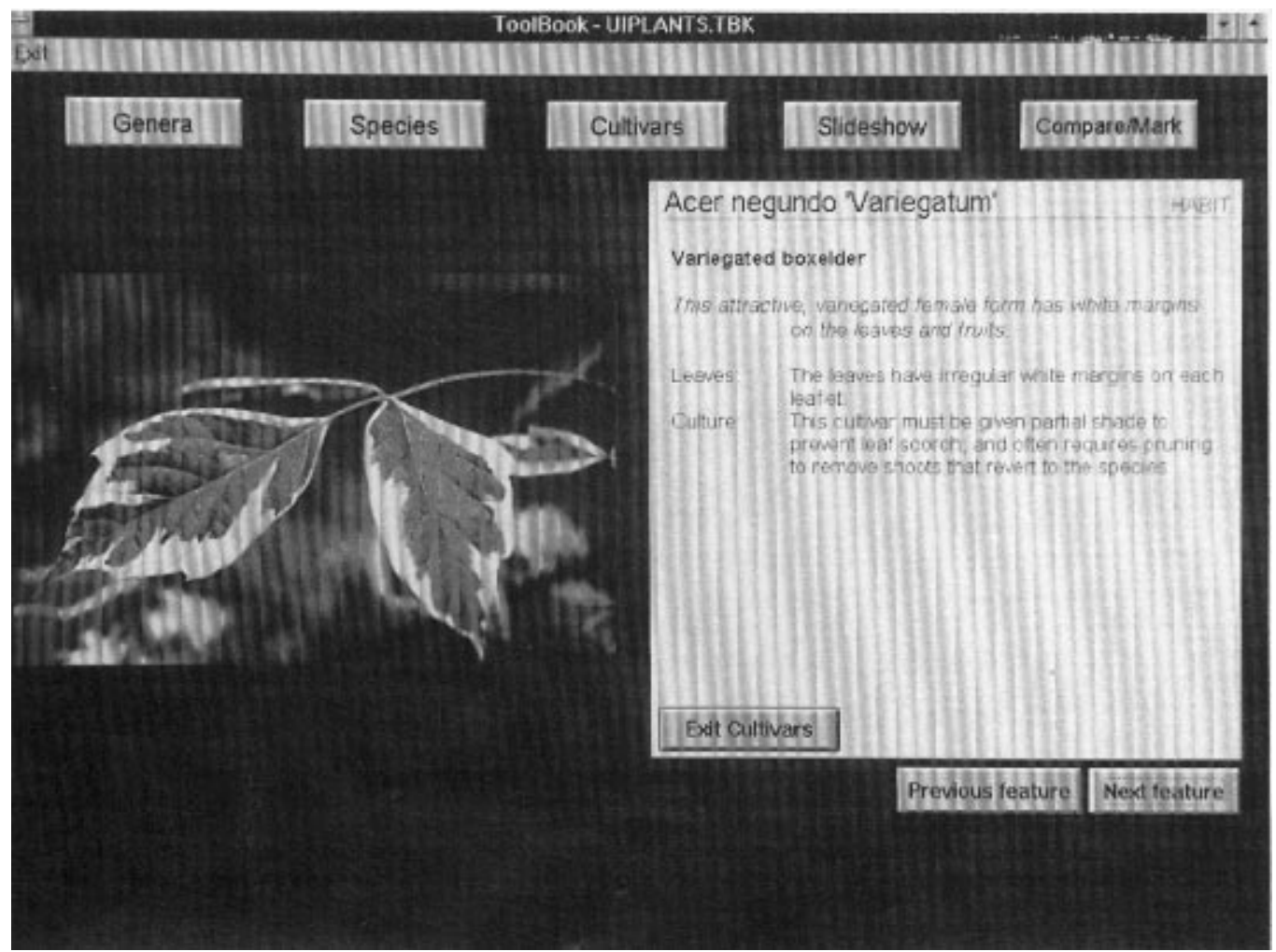

The main portion of UIPLANTS is encyclopedic in nature, displaying the full-color images and text side by side for each plant characteristic. The graphical nature of the program makes it simple to switch between characteristics, and it does not overwhelm the user with too manyoptions. Genera, species, and cultivars can be selected by a click of the mouse on scrollable lists of choices. The only computer background required for usersis theability to usea mouseto point and click on items. tivar is selected with the mouse, an imageof theplant

Fig. 2. Sample screen displaying information on a cultivar.
When a species or cul- 


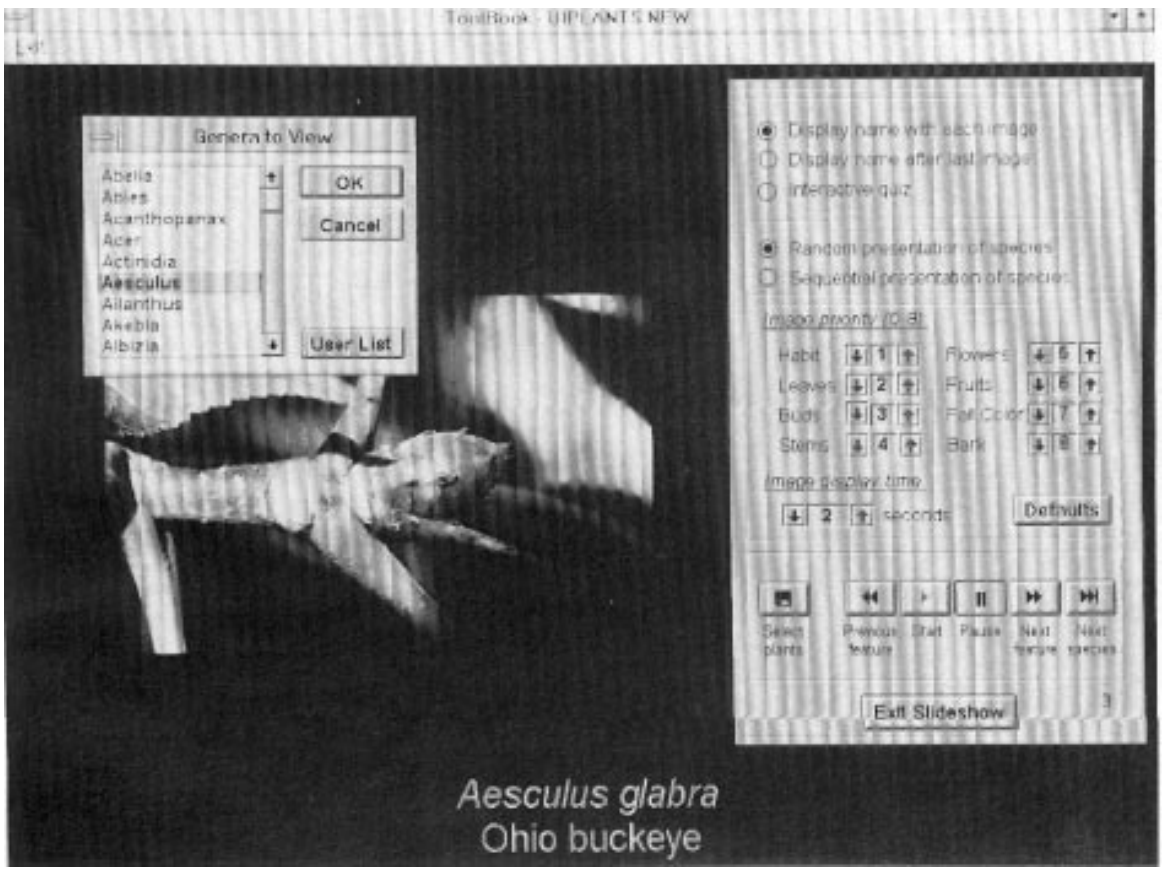

Fig. 3. Sample slideshow screen displaying user selectable options.

habit appears, accompanied by text describing the habit, size, color, texture, hardiness, and native habitat (Fig. 1). In most cases, multiple images are available for each selected feature, allowing users to see diversity in each plant feature. For example, a user often would be able to see young and more mature plant specimenswhen looking at a plant's habit. The inclusion of multiple images for each feature also providesan opportunity for the authorsto show arange of characteristics, such as the variation that commonly occurs in habit or leaf shape or an average flowering display versus an extraordinary one. It also helps to avoid problems with students' associating an identification with some extraneousbackground image, such as "Oh! That's the viburnum with the red car in the background!"

The program revolves around the species page, from which all other featuresareavailableasbutton choices. U sers can go to any feature such as leaves, buds, stems, flowers, fruit, fall color, bark, and culture in a nonlinear fashion. The compare/ mark page, slideshow, and cultivar fea- tures are also available from the species screen. When the cultivar button is chosen, a list of cultivars appears for the species the user is currently viewing. Cultivars are selected in a manner similar to species and presentations begin with a habit or other image of the main distinguishing feature (Fig. 2). At the touch of thenext feature button, the viewer ispresented with other features of the cultivar.

Stideshow. The slideshow portion of this program displays a sequence of plant
Fig. 4. Sample screen displaying the side-by-side format of the compare feature.

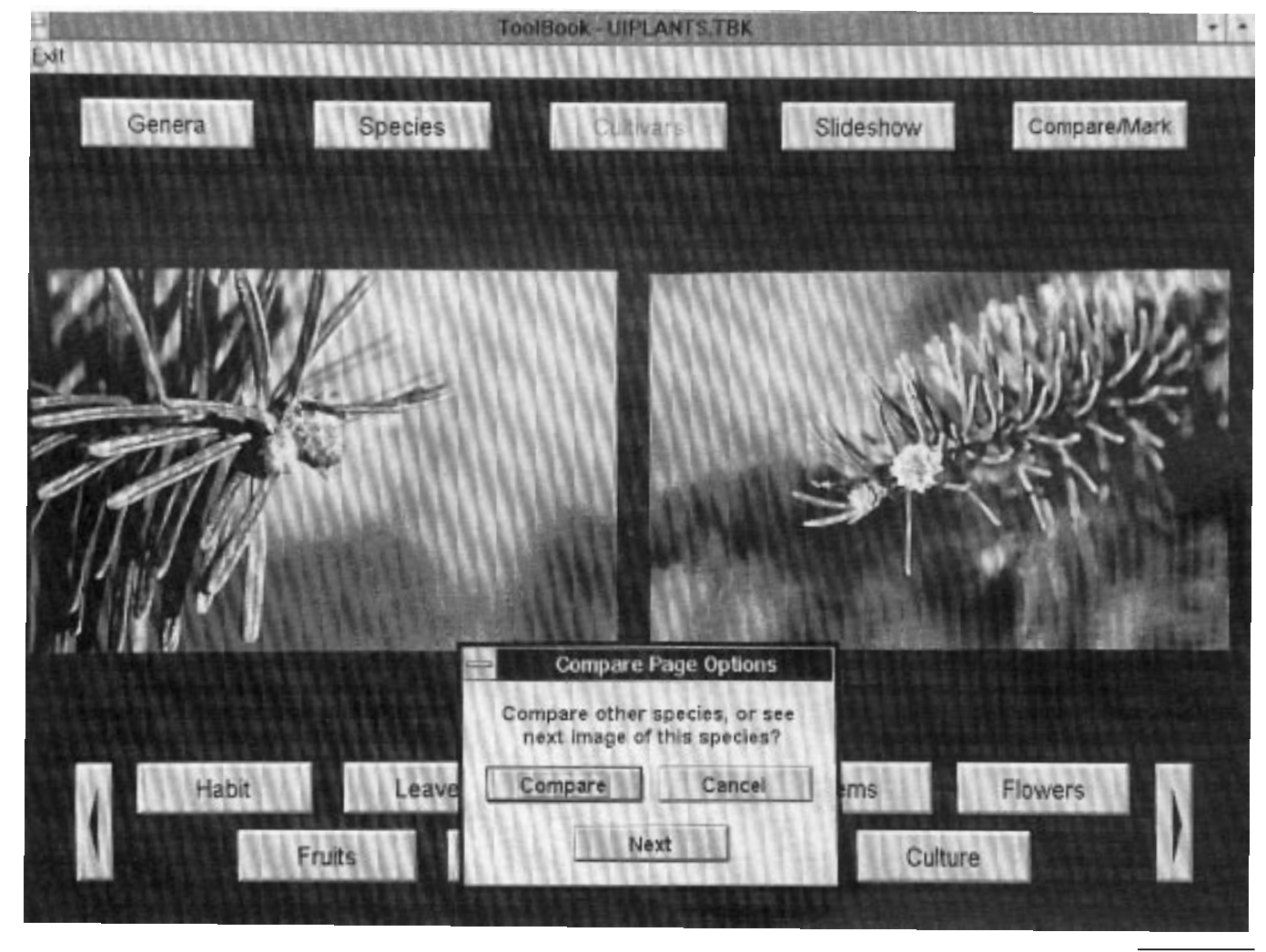


imageswithout theaccompanying text. G reat user flexibility in choice of optionsmakes this a powerful addition to the program. U sers choose the images they see, the order in which they are presented, and the duration of each image on the screen. All species images in the database are available in the slideshow and can be presented in either a sequential or random fashion. $U$ sers can pause the show at any time or change the display options, even while the slideshow is in progress. The slideshow also contains a routine that randomly selects from among the available images for each chosen plant feature, resulting in a different selection of images each timethe slideshow is run.

When the slideshow button is pressed, a menu appears prompting the user to determine which single genus or multiple genera are to be displayed. In addition, the program allows for the selection of custom user-defined lists that an instructor, landscape designer, or other professional might set up in advance. By adding the names of desired species to a text file, lists of plants can be created for viewing in the slideshow. I nstructors can group plants by week, semester, genus, flowering time, or any characteristic that aids in presenting the material to students. T rades people can make lists of plants for designs to show potential clients or simply to show customers plants that they sell.

$L$ andscape contractorsand designersmay make customized lists to show potential clients plant materials they have selected for their landscapedesign. They can choose which images they wish to show and in what order. If you were displaying A ronia arbutifolia, red chokeberry, to a client, you might want the fruit displayed first, followed by the flowers and fall color. And if one fruit image were better than another, you would want to have the program to use the superior image every time. This is achieved by cycling through the images in a preprogrammed browser and selecting the images you want displayed. Student users often choose to select plant groups corresponding to the lists covered in laboratories. These lists allow the viewer to concentrate on species that are of immediate concern. At the U niv. of I llinois, the customized lists include plants grouped by lab dates and semester. C ustom lists used at the C ollege of Lake County allow the instructor to select a different list of plants.

O nce the genera or species have been chosen, the user is asked if he wantsto see the plant names with each image in a time-delayed fashion or if he wants to take an interactive quiz (Fig. 3). Thesecond option allows students a short time to identify the species before seeing the name. In the quiz format, the screen displays the first image of the randomly chosen species and asks the user to correctly spell the scientific name of the plant. The student can respond by typing in the answer or looking at additional features to provide more clues. Visuals from all of the prechosen categories are available to help identify the plant. If the response is not correct, the software tells the user to try again, but gives the answer after three wrong responses. The program doesnot score or log data on student results.

After selecting the name display options, the user can then choose the display order of the habit, leaves, buds, stems, flowers, fruit, fall color, and bark for the genera they have picked (Fig. 3). Students studying for an outdoor identification test might decide to view only the habits, leaves, buds, stems, and bark, whereas habit, flowers, fruits, fall color, and bark might be used to prepare for a lecture examination.

The length of the display time for each feature can be set within a range of 2 to 60 seconds. The pause button can be pressed at any time to allow additional viewing time, or the display time can be changed while the slide show is in progress.

The user can exit slideshow at any time by a pressing the exit slideshow button. U pon exiting the slideshow, users are returned to the page they were viewing before selecting the slideshow option.

Compare and mark page. U I PLANTS offers several options through the compare/ mark page button. This button brings up a menu that allows a user to mark a page for review later or compare any image with one of those already marked or with any of the other 8000 images in the program. The two chosen images are displayed side by side on the monitor, allowing for careful comparison. I n thismanner, studentsor professionals may compare leaves or any other feature of a closely related plant side by side (Fig. 4) so the differences can be studied. These comparisons are difficult to accomplish with standard printed references.

\section{Availability and future development}

U IPL AN TS will be available through a commercial software publishing company in late 1996. D etails on the publisher and product price are not available. A UIPLANTS homepage is available on the World Wide Web at http:/ / www.mallorn. com/ uiplants. U IPLANTS is being upgraded continually to meet new user needs. Although the existing program features are more than adequate for the average user, there are still some things that we would like to see in future releases. These include 
- adding sound for pronunciation of scientific names,

- incorporating artificial intelligencein the quiz portion of the slideshow, telling the user why an answer was wrong,

- RTF or HTML support for text,

- access to plants via common names,

- adding more images and cultivars,

- marketing ageneric shell with adeveloper's package to allow purchasers to create their own program for other plant materials or any other educational topic, and

- printing capabilities

\section{Literature Cited}

Dirr, M.A. 1990. M anual of woody landscape plants. 4th ed. Stipes, Champaign, III.

Flint, H.L. 1983. Landscape plants for eastern $\mathrm{N}$ orth America exclusive of F lorida and the I mmediate Gulf Coast. Wiley, N ew York.

Phillips, R and M.R ix. 1989. The Random H ouse book of shrubs. Random H ouse, N ew York.

Phillips, R. 1978. The Random H ouse book of trees of $\mathrm{N}$ orth America and Europe. Random $\mathrm{H}$ ouse, $\mathrm{N}$ ew York.

Wyman, D. 1990. Trees for American gardens. 3rd ed. M acmillan, N ew York. 